\title{
Pharmacological modulation of oncogenic Ras by natural products and their derivatives: renewed hope in the discovery of novel anti-Ras drugs
}

\begin{abstract}
Oncogenic rat sarcoma (Ras) is linked to the most fatal cancers such as those of the pancreas, colon, and lung. Decades of research to discover an efficacious drug that can block oncogenic Ras signaling have yielded disappointing results; thus, Ras was considered "undruggable" until recently. Inhibitors that directly target Ras by binding to previously undiscovered pockets have been recently identified. Some of these molecules are either isolated from natural products or derived from natural compounds. In this review, we described the potential of these compounds and other inhibitors of Ras signaling in drugging Ras. We highlighted the modes of action of these compounds in suppressing signaling pathways activated by oncogenic Ras, such as mitogen-activated protein kinase (MAPK) signaling and the phosphoinositide-3-kinase (PI3K) pathways. The anti-Ras strategy of these compounds can be categorized into four main types: inhibition of Ras-effector interaction, interference of Ras membrane association, prevention of Ras-guanosine triphosphate (GTP) formation, and downregulation of Ras proteins. Another promising strategy that must be validated experimentally is enhancement of the intrinsic Ras-guanosine triphosphatase (GTPase) activity by small chemical entities. Among the inhibitors of Ras signaling that were reported thus far, salirasib and TLN-4601 have been tested for their clinical efficacy. Although both compounds passed phase I trials, they failed in their respective phase II trials. Therefore, new compounds of natural origin with relevant clinical activity against Ras-driven malignancies are urgently needed. Apart from salirasib and TLN-4601, some other compounds with a proven inhibitory effect on Ras signaling include derivatives of salirasib, sulindac, polyamine, andrographolide, lipstatin, levoglucosenone, rasfonin, and quercetin.
\end{abstract}

Keyword: Oncogenic Ras; Anti-Ras; Cancer; Natural products; Derivatives; Strategy 